\title{
Idade - Deve ser levada em conta? Estudo da morbidade perioperatória e a evolução no longo prazo dos pacientes acima de 70 anos submetidos à cirurgia da coluna vertebral, devido a doenças degenerativas lombares*
}

\section{Age - Does it Really Count? A Study of the Perioperative Morbidity and Long-Term Outcome in Patients Above 70 Years of Age Undergoing Spine Surgery for Lumbar Degenerative Disorders}

\author{
Nigil Sadanandan Palliyil ${ }^{1}$ Siddharth Shah ${ }^{1} \quad$ Ravi Ranjan Rai $^{1}$ Samir Dalvie ${ }^{1}$ Joseph Monteiro ${ }^{1}$ \\ ${ }^{1}$ Departamento de Ortopedia, P. D. Hinduja National Hospital e \\ Medical Research Centre, Mumbai, India \\ Rev Bras Ortop 2020;55(3):298-303. \\ Endereço para correspondência Nigil Sadanandan Palliyil, DNB, \\ Department of Orthopaedics, FNB Spine Fellow, P. D. Hinduja National \\ Hospital and Medical Research Centre, Veer Savarkar Marg, Mahim, \\ Mumbai, 400016, India (e-mail: nigilanand@hotmail.com).
}

\section{Resumo \\ Palavras-chave \\ - cirurgia da coluna \\ - degeneração do disco intervertebral/ cirurgia \\ - vértebras lombares/ cirurgia \\ - idosos}

Objetivo Avaliar o resultado no longo prazo e a morbidade perioperatória em cirurgias da coluna vertebral, devido a doenças lombares degenerativas e, assim, avaliar a segurança da cirurgia na população idosa.

Métodos Estudo retrospectivo de pacientes com idade superior a 70 anos, submetidos à cirurgia em virtude de distúrbios lombares degenerativos, entre 2011 e 2015. Foram avaliados os dados demográficos, clínicos e cirúrgicos dos pacientes; comorbidades; complicações perioperatórias; escores de dor no pré e no pós-operatório; índice de incapacidade de Oswestry (ODI, na sigla em inglês); satisfação do paciente e a mortalidade geral.

Resultados Foram estudados 103 pacientes (homens:mulheres, 55:48) com idade média de 74,6 anos (70 a 85 anos). 60 pacientes (58,2\%) apresentaram somente descompressão, enquanto $43(41,8 \%)$ apresentaram descompressão e fusão. O tempo médio de internação foi de 5,7 dias. O tempo médio de acompanhamento foi de 47,6 meses (24-73 meses). Os pacientes relataram melhora significativa da dor nas costas (pontuação numérica da dor 7,7 versus 1,$6 ; p<0,001$ ), dor nas pernas (pontuação numérica da dor 7,4 versus 1,$7 ; p<0,001$ ), incapacidade (ODI 82,3 versus 19,1; $p<0,001)$ e distância percorrida a pé $(p<0,001)$. Um total de $76 \%$ dos pacientes estavam satisfeitos com os resultados no momento do acompanhamento final. 26 pacientes $(25,24 \%)$ apresentaram complicações perioperatórias, todas sem relevância e sem mortalidade. As complicações intra e pós-operatórias mais comuns foram ruptura dural $(6,79 \%)$ e infecção do trato urinário $(6,79 \%)$, respectivamente.

\footnotetext{
Estudo realizado pelo Departamento de Ortopedia, P.D Hinduja National Hospital e Medical Research Center, Mumbai, Índia.
}

recebido

28 de Setembro de 2018 aceito

05 de Fevereiro de 2019
DOI https://doi.org/ $10.1055 / \mathrm{s}-0039-1700833$. ISSN $0102-3616$
Copyright $\odot 2020$ by Sociedade Brasileira License terms de Ortopedia e Traumatologia. Published by Thieme Revinter Publicações Ltda, Rio de Janeiro, Brazil 


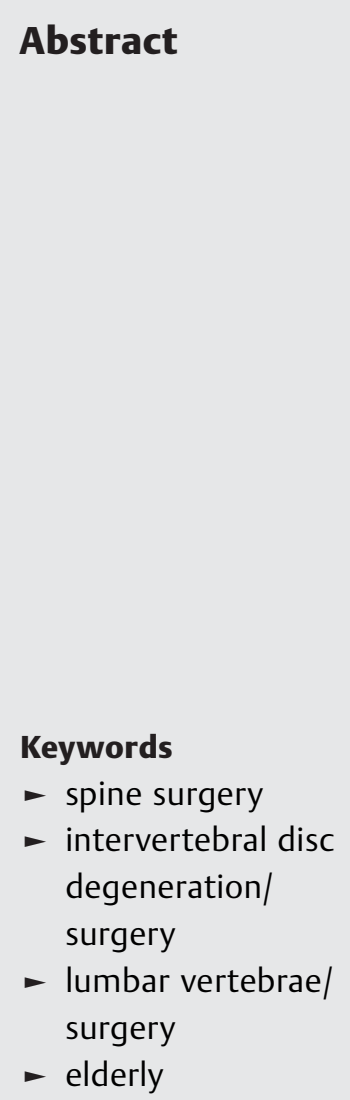

Conclusões Com meticulosos cuidados perioperatórios, a cirurgia da coluna lombar é segura e eficaz na população idosa. Os pacientes tiveram um maior tempo médio de internação hospitalar, em virtude do programa de reabilitação gradual e abrangente. A presença de comorbidades ou complicações perioperatórias sem relevância, não aumentou a morbidade geral, nem afetou os resultados clínicos da cirurgia em nosso estudo.

Objective To assess the long-term outcome and perioperative morbidity in spine surgeries for lumbar degenerative disorders and, thereby, to evaluate the safety of surgery in the aging population.

Methods Retrospective study of patients aged $>70$ years, operated for degenerative lumbar disorders between 2011 and 2015. We evaluated patient demographic, clinical and surgical data; comorbidities, perioperative complications, pre \& postoperative pain scores and Oswestry disability index (ODI) scores, patient satisfaction and overall mortality.

Results A total of 103 patients (Males: Females55:48) with mean age 74.6 years (70-85yrs) were studied. 60 patients (58.2\%) had decompression alone, while 43 (41.8\%) had decompression \& fusion. Mean hospital stay was 5.7days. Mean follow-up was 47.6 months (24-73mnths). Patients reported significant improvement in backpain (Numerical pain score 7.7 vs $1.6 ; p<0.001$ ), leg pain (Numerical pain score 7.4 vs $1.7 ; p<0.001$ ), disability (ODI 82.3 vs $19.1 ; p<0.001$ ) and walking distance $(p<0.001) .76 \%$ patients were satisfied with the results at the time of final followup. 26 patients $(25.24 \%$ ) had perioperative complications which were all minor, without mortality. Most common intraoperative \& postoperative complications were dural tear $(6.79 \%) \&$ urinary tract infection $(6.79 \%)$ respectively.

Conclusions With meticulous perioperative care lumbar spine surgery is safe and effective in elderly population. Patients had longer mean hospital stay in view of the gradual and comprehensive rehabilitation program. Presence of comorbidities or minor perioperative complications did not increase the overall morbidity or affect the clinical outcomes of surgery in our study.

\section{Introdução}

A proporção da população idosa em todo o mundo cresceu de $<1 \%$ em 1900 para 6,2\% em 1992. Prevê-se uma expansão da população idosa global para $20 \%$ até o ano de $2020 .{ }^{1} \mathrm{Na}$ Índia, de acordo com o censo populacional, existem quase 104 milhões de idosos (com 60 anos ou mais). Verificou-se que a causa mais comum de incapacidade entre os idosos é a locomotora e a visual. ${ }^{1}$ O distúrbio degenerativo da coluna lombar é uma das principais causas da disfunção locomotora, levando a uma significativa perda de função e incapacidade para realizar atividades da vida diária.

Muitas dessas doenças degenerativas lombares (DDLs) justificam a intervenção cirúrgica, a fim de melhorar a qualidade de vida da população idosa. No entanto, controvérsias significativas envolvem a relação risco-benefício nas cirurgias da coluna nesse grupo etário, principalmente, em virtude das comorbidades existentes devido ao processo de envelhecimento. Os pacientes e familiares costumam ficar apreensivos e preocupados com a segurança da cirurgia da coluna lombar em idosos.

A revisão da literatura demonstrou resultados conflitantes em relação ao desfecho do tratamento cirúrgico da DDL na população idosa. ${ }^{2-5}$ Demograficamente, cada população apresenta variações em termos de suscetibilidade a DDL, os estudos e os dados dos resultados disponíveis são escassos no subcontinente indiano.

O objetivo deste estudo foi avaliar a morbidade perioperatória e determinar o resultado e a satisfação no longo prazo dos pacientes com idade superior a 70 anos, submetidos à cirurgia da coluna vertebral em função da DDL.

\section{Materiais e Métodos}

\section{Escolha do Tema}

Este foi um estudo retrospectivo com dados coletados prospectivamente de todos os casos consecutivos e contínuos (103 pacientes) de cirurgia da coluna lombar, realizados entre janeiro de 2011 e dezembro de 2015, em razão de distúrbios degenerativos da coluna vertebral, em pacientes com idade superior a 70 anos. Foi obtida a aprovação do Conselho de Revisão Institucional. Todas as cirurgias foram realizadas na instituição dos autores pelo autor sênior. Os pacientes que compareciam à instituição clínica apresentando sintomas persistentes de dor nas costas ou nas pernas, claudicação neurogênica e/ou radiculopatia eram submetidos a uma ressonância 
magnética, a fim de confirmar o diagnóstico de DDL. Pacientes que apresentavam outras patologias, tais como tumor, trauma, ou infecção, foram excluídos do estudo. Todos os pacientes foram submetidos a um estudo inicial, por meio de um tratamento conservador durante um período de 6 a 12 semanas. $\mathrm{O}$ tratamento incluiu analgésicos, modificação das atividades, reabilitação física, através de um programa de fortalecimento lombar e central. Ao final das 12 semanas, era oferecida uma solução cirúrgica apenas para aqueles pacientes com sintomas incapacitantes e persistentes. A decisão de adição de fusão era baseada na presença ou ausência de instabilidade vertebral demonstrável. Foram utilizados na avaliação da instabilidade os critérios radiológicos de Punjabi. ${ }^{6}$

Todos os casos de fusão foram instrumentados com a fixação posterior de parafusos pediculares. O autoenxerto colhido localmente foi utilizado em todos os casos, a partir de processos espinhosos vertebrais e da lâmina. Dos 103 pacientes, $60(58,2 \%)$ foram submetidos somente à descompressão, enquanto 43 (41,8\%) foram submetidos à descompressão e fusão. Desses últimos, 21,5\% dos pacientes foram submetidos à fusão intervertebral lombar transforaminal (TLIF, na sigla em inglês), enquanto 20,3\% apresentaram fusão intersomática lombar posterior (PLF, na sigla em inglês).

\section{Avaliação do Paciente}

Os dados demográficos dos pacientes foram registrados e a avaliação da morbidade perioperatória foi realizada com todos os pacientes, a partir de gráficos e prontuários médicos. Os parâmetros clínicos estudados incluíram a classificação American Society of Anaesthesiologists (ASA) do estado físico, comorbidades médicas pré-operatórias, duração da cirurgia, complicações médicas e cirúrgicas perioperatórias, além do tempo de internação. O período de acompanhamento variou de 2 a 6 anos (média de 47,6 meses). A avaliação do resultado clínico foi realizada no acompanhamento final da conclusão do período estudado (dezembro de 2017), por meio de entrevista telefônica. Um total de $84(81,55 \%)$ pacientes estavam disponíveis para o acompanhamento final: 9 pacientes faleceram devido a causas não relacionadas à cirurgia, e 10 pacientes foram perdidos durante o acompanhamento. Os pacientes responderam a um conjunto pré-definido de perguntas, a fim de que pudesse ser avaliado o resultado clínico e a satisfação, tendo sido registradas todas as respostas. As medidas dos resultados avaliados no questionário incluíram escores numéricos, com a finalidade de medir a incapacidade e avaliar o status ambulatorial, em função de dores nas costas e nas pernas, por meio do índice de incapacidade Oswestry (ODI) modificado. Os escores de dor foram medidos usando a escala numérica de classificação de dor, de 0 a 10. O ODI foi utilizado para avaliar as atividades da vida diária. O status ambulatorial de cada paciente foi avaliado, através do registro da distância pré-operatória e pós-operatória, que os pacientes podiam caminhar antes de sentir dor claudicante ou radicular nas extremidades. Foi registrada também a satisfação subjetiva geral do paciente, em que foi perguntado se estavam "satisfeitos", "insatisfeitos" ou "indiferentes", com os resultados da cirurgia. Os parâmetros do desfecho clínico pré-operatório foram comparados com os obtidos no momento da entrevista no acompanhamento final.

\section{Análise Estatística}

A análise dos dados foi realizada utilizando o programa SPSS versão 16.0 (SPSS Inc, Chicago, IL, EUA). Os dados foram expressos em faixa de valores, média e/ou desvio padrão. $\mathrm{O}$ teste estatístico de Wilcoxon (Wilcoxon signed ranks test) foi utilizado para análise e comparação dos parâmetros pré e pós-operatórios. Os dados descritivos foram expressos como porcentagens calculadas do total.

\section{Resultados}

Foram revisados os prontuários de 103 pacientes ( 55 homens $\mathrm{e}$ 48 mulheres). A idade média da população do estudo no momento da cirurgia era de 74,62 anos (70-85 anos). 0 acompanhamento médio após a cirurgia foi de 47,6 meses (24-73 meses) (-Tabela 1). Os diagnósticos pré-operatórios incluíram estenose do canal lombar (devido à hipertrofia do ligamento flavum e artropatia facetária) em 61 pacientes (59,2\%), espondilolistese degenerativa em 34 pacientes (33\%), e instabilidade pós-operatória em 8 pacientes (7,7\%). Os déficits neurológicos pré-operatórios, incluindo os sensoriais e motores ou uma combinação de ambos, estavam presentes em 46 pacientes (44,66\%). A duração média da dor nas costas e nas pernas antes da cirurgia era de 11,12 meses e 6,51 meses, respectivamente.

Uma condição comórbida estava presente em pelo menos $76,6 \%$ dos pacientes. O número médio de comorbidades por paciente era de 1,56 (-Tabela 2). A hipertensão (52,4\%) foi a comorbidade mais comum, seguida por diabetes mellitus relatado em $41,7 \%$ dos pacientes. Os hipoglicemiantes orais eram utilizados por $74,2 \%$ dos pacientes, enquanto $25,8 \%$ faziam uso de insulina. A doença isqueêmica do coração (DIC) foi encontrada em $17,4 \%$ dos pacientes, dos quais $9,2 \%$ foram submetidos à angioplastia no passado, enquanto $6,2 \%$ foram submetidos à cirurgia de revascularização do miocárdio (CRM), e os $2 \%$ restantes foram tratados com medicamentos. Todos esses pacientes estavam fazendo uso de drogas antiplaquetárias. Elas foram suspensas cinco dias antes da cirurgia e reiniciadas a partir do segundo dia do pós-operatório, após a remoção do dreno cirúrgico.

No pré-operatório, o status físico do paciente foi classificado em graus, variando de I a VI de acordo com o sistema de classificação da ASA (-Tabela 3). A maioria dos pacientes (66\%) foi classificada como grau III, de acordo com o sistema

Tabela 1 Dados demográficos dos 103 idosos submetidos à cirurgia

\begin{tabular}{|l|l|}
\hline Idade Média & 74,62 \\
\hline Sexo (Masculino/feminino) & $55 / 48$ \\
\hline ASA (I/II/III) & $6,7 \% ; 66 \% ; 27,3 \%$ \\
\hline Média de acompanhamento & 47,6 months \\
\hline $\begin{array}{l}\text { Óbitos (no momento do } \\
\text { acompanhamento final) }\end{array}$ & 9 \\
\hline
\end{tabular}


Tabela 2 Distribuição das comorbidades

\begin{tabular}{|l|l|}
\hline Comorbidade & Número de Pacientes \\
\hline Hipertensão & 54 \\
\hline Diabetes & 43 \\
\hline Cardiopatia isquêmica & 18 \\
\hline Asma/DPOC & 7 \\
\hline Disfunção tireoidiana & 6 \\
\hline Doença renal crônica & 4 \\
\hline Parkinsonismo & 4 \\
\hline Tuberculose & 4 \\
\hline Malignidade & 3 \\
\hline Artrite reumatoide & 1 \\
\hline Espondilite anquilosante & 1 \\
\hline Depressão & 1 \\
\hline
\end{tabular}

Abreviatura: DPOC, doença pulmonar obstrutiva crônica.

Tabela 3 Classificação da American Society of Anesthesiologists do estado físico

\begin{tabular}{|l|l|}
\hline Classe & Definição \\
\hline I & Nenhuma doença sistêmica \\
\hline II & Doença sistêmica leve a moderada \\
\hline III & Doença sistêmica grave \\
\hline IV & Doença sistêmica grave com risco de vida \\
\hline V & $\begin{array}{l}\text { Paciente moribundo com poucas } \\
\text { chances de sobrevivência }\end{array}$ \\
\hline
\end{tabular}

ASA, enquanto $27,3 \%$ e $6,7 \%$ dos pacientes foram classificados como grau III e grau I, respectivamente.

O tempo médio de duração da cirurgia foi de 136,94 minutos. Como previsto, a duração da cirurgia no grupo descompressão foi menor (120,4 minutos), em comparação com o grupo de fusão (144,6 minutos). Dentro do grupo de fusão, o TLIF consumiu mais tempo em comparação com o PLF (159,1 minutos versus 130,2 minutos, respectivamente). A perda de sangue durante a cirurgia foi em média de $346,6 \mathrm{ml}$, sendo maior no grupo de fixação do que no grupo somente de descompressão (396,2 $\mathrm{ml}$ versus $306,3 \mathrm{ml}$, respectivamente). A transfusão foi necessária no perioperatório em 14 pacientes (13,5\%), em virtude de terem apresentado uma perda sanguínea média intraoperatória de $804,16 \mathrm{ml}$. Foi necessária a internação de 7 pacientes $(6,75 \%)$ em unidade de terapia intensiva (UTI) pós-operatória: 2 pacientes desenvolveram arritmia cardíaca no pós-operatório, 1 apresentou colecistite aguda, enquanto os outros 4 pacientes já apresentavam um histórico prévio de doenças cardíacas, com comprometimento da reserva cardíaca, sendo mantidos em UTI para monitoramento e observação.

No geral, foram observadas complicações perioperatórias em 26 dos 103 pacientes operados (25,24\%). Nove pacientes (8,73\%) apresentaram complicações intraoperatórias: lesão dural em 7 pacientes (6,79\%), e hipotensão e arritmia em 2
Tabela 4 Complicações perioperatórias

\begin{tabular}{|l|l|}
\hline Complicações & Número (\%) \\
\hline Ruptura dural & $7(6,79 \%)$ \\
\hline Infecção do sítio cirúrgico & $3(2,91 \%)$ \\
\hline Infeç̧ão do trato urinário & $7(6,79 \%)$ \\
\hline Sensório alterado & $3(2,91 \%)$ \\
\hline Náuseas/vômitos & $3(2,91 \%)$ \\
\hline Hipotensão/arritmia & $2(1,94 \%)$ \\
\hline Colecistite aguda & $1(0,97 \%)$ \\
\hline Total & $26(25,24 \%)$ \\
\hline
\end{tabular}

pacientes (1,94\%). Dos sete pacientes com ruptura dural incidental, quatro foram submetidos à cirurgia revisional da coluna vertebral, sendo realizada uma cirurgia anterior da coluna no mesmo nível/adjacente. O vazamento dural foi selado com enxerto de gordura autólogo. 0 reparo dural primário não foi realizado em nenhum dos casos. Foi inserido um dreno subfascial em todos os pacientes, o qual foi mantido sem carga. Os pacientes foram mantidos em repouso rigoroso no leito por $3 \mathrm{a}$ 5 dias, até que nenhuma evidência de líquido cefalorradiquiano (LCR) fosse observada no dreno, após foram gradualmente mobilizados e posteriormente o dreno foi removido. Não foram observadas complicações residuais relacionadas ao vazamento dural. Foram observadas complicações pós-operatórias em 18,4\% dos pacientes, complicações médicas em 15,5\%, e complicações cirúrgicas em 2,91\%. A infecção do trato urinário $(6,79 \%)$ foi a complicação médica mais comum, seguida de diseletrolitemia e sensório alterado (2,91\%), além de vômitos recorrentes (2,91\%) ( - Tabela 4). Ocorreu infecção superficial da ferida cirúrgica em 3 pacientes $(2,91 \%)$. Todos os casos foram tratados com um curso prolongado de antibióticos e curativos periódicos. Embora a cicatrização da ferida tenha sido retardada, nenhum desses casos exigiu desbridamento secundário ou ressutura. Não houve mortalidade perioperatória ou complicações com risco de vida em nosso estudo.

A presença de comorbidades médicas não foi uma causa direta de complicações cirúrgicas ou clínicas em nenhum de nossos pacientes. Na análise comparativa dos subgrupos, observamos que não houve aumento no percentual de complicações, em pacientes pertencentes aos graus mais elevados do sistema de classificação ASA, em comparação com aqueles que receberam grau menos elevado no mesmo sistema ASA (- Tabela 5), tornando o sistema de classificação ASA uma medida de desfecho menos confiável para prever complicações pós-operatórias.

O tempo médio de internação foi de 5,69 dias. A duração da internação hospitalar foi prolongada, já que os pacientes idosos não puderam tolerar uma fisioterapia agressiva, diferentemente da população mais jovem. Portanto, foi realizada a fisioterapia gradual, começando com exercícios no leito, progredindo para a mobilização com o andador e, finalmente, tornando-os capazes e confiantes na mobilização independente. Todos os pacientes receberam alta, permanecendo em suas residências. Nenhum dos pacientes recebeu alta para 
Tabela 5 Agrupamento das complicações relevantes para ASA

\begin{tabular}{|l|l|l|l|}
\hline ASA & I & II & III \\
\hline Número de pacientes & 7 & 68 & 28 \\
\hline Complicações & \multicolumn{4}{|l|}{} \\
\hline Ruptura dural & 0 & 6 & 1 \\
\hline Infecção do sítio cirúrgico & 0 & 2 & 1 \\
\hline Infecção do trato urinário & 2 & 4 & 1 \\
\hline Sensório alterado & 0 & 2 & 1 \\
\hline Vômitos & 1 & 1 & 1 \\
\hline Hipotensão/arritmia & 0 & 1 & 1 \\
\hline Colecistite aguda & 0 & 1 & 0 \\
\hline $\begin{array}{l}\text { Número total de } \\
\text { complicações }\end{array}$ & 3 & $\begin{array}{l}17 \\
(25 \%)\end{array}$ & $\begin{array}{l}6 \\
(21,4 \%)\end{array}$ \\
\hline
\end{tabular}

Abreviatura: ASA, American Society of Anesthesiologists.

asilos, centros de reabilitação ou unidades de atendimento ambulatorial.

O tempo de internação foi maior nos pacientes submetidos à descompressão e fusão (6,16 dias), quando comparados aos pacientes submetidos somente à descompressão (5,35 dias). Para os pacientes que tiveram ruptura dural iatrogênica incidental intraoperatória, o tempo de internação aumentou para uma média de 8,71 dias. Devido a mortes e a relutância em participar, apenas $84(81,55 \%)$ pacientes estavam disponíveis para avaliação final de acompanhamento dos resultados clínicos. No pré-operatório, os escores numéricos médios de dor nas pernas e nas costas relatados foram 7,37 $\pm 0,9$ e 7,6 $\pm 0,8$, respectivamente. Os escores numéricos médios de dor caíram para $1,57 \pm 1,7$ e $1,56 \pm 1,9$, respectivamente, no acompanhamento final após a cirurgia, sugerindo melhora significativa $(p<0,0001)$ ( - Tabela 6$)$. O escore médio do ODI melhorou de $82,32 \%$ no pré-operatório para $19,17 \%$ no acompanhamento final, uma melhora altamente significativa $(p<0,0001)$ (-Tabela 6). 0 estado ambulatorial de $84,52 \%$ dos pacientes havia melhorado, em pelo menos um grau em relação ao valor pré-operatório, no momento do acompanhamento final (-Fig. 1). Durante o acompanhamento, 76\% dos pacientes estavam satisfeitos com a cirurgia, enquanto $13 \%$ dos pacientes estavam insatisfeitos, e $11 \%$ dos pacientes se mostravam indiferentes.

No momento do acompanhamento final, 9 pacientes morreram devido a causas não relacionadas à cirurgia, sendo a duração média entre a cirurgia e o óbito de 45,1 meses. No entanto, nenhum paciente faleceu no pós-operatório ime-

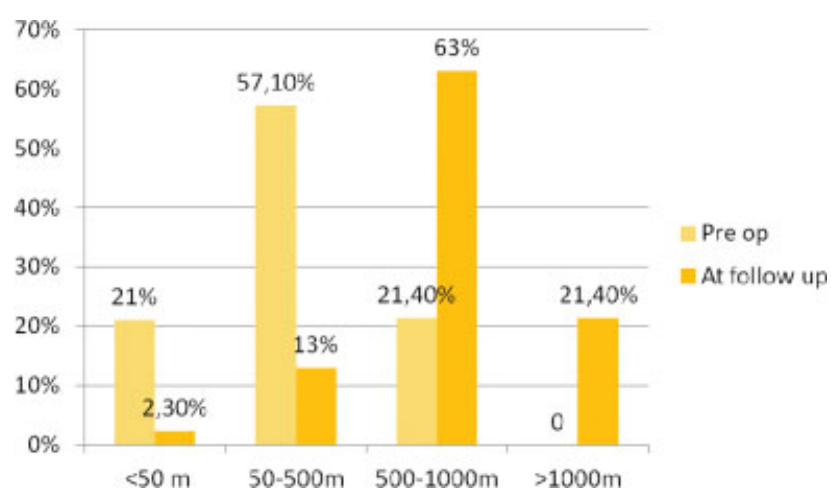

Fig. 1 Curta distância dos pacientes no pré-operatório e no momento do acompanhamento final no pós-operatório.

diato ( $<3$ meses). Nenhum paciente necessitou de cirurgia revisional da coluna lombar até o tempo médio de acompanhamento que foi de 47,6 meses.

\section{Discussão}

Com o avanço significativo da medicina moderna, com o aumento da expectativa de vida da população em todo o mundo, o envelhecimento e a degeneração andam de mãos dadas, levando a um aumento no número de distúrbios degenerativos da coluna lombar, muitos deles justificando tratamento cirúrgico. Este estudo retrospectivo investigou 103 casos consecutivos de pacientes com idade superior a 70 anos, com DDLs que foram submetidos à cirurgia da coluna vertebral. A morbidade perioperatória geral, o desfecho clínico no longo prazo e a satisfação do paciente foram favoráveis nesta população do estudo.

O padrão de comorbidade em nosso estudo foi comparável ao relatado por Ragab et al. ${ }^{5}$ e Shabat et al. ${ }^{7}$ Nem a presença de comorbidades nem o grau ASA de classificação de pacientes, tiveram influência na incidência de complicações perioperatórias ou no resultado geral.

Embora não tenham sido observadas complicações importantes, foram encontradas pequenas complicações relacionadas à cirurgia em $25,24 \%$ dos pacientes. Ragab et al., ${ }^{5}$ em seu estudo com 118 pacientes (idade média de 74 anos e acompanhamento médio de 84 meses) submetidos a cirurgias da coluna lombar, relataram uma taxa de complicação de $20 \%$.

Quatro dos sete pacientes com ruptura dural em nosso estudo foram submetidos á cirurgia revisional da coluna. Smorgick et al. $^{8}$ observaram uma incidência significativamente maior de ruptura dural na cirurgia revisional da coluna vertebral (29/116 pacientes; 25\%). Canal mais estreito, flavum

Tabela 6 Medidas de desfechos clínicos

\begin{tabular}{|l|l|l|l|l|l|}
\hline & \multicolumn{2}{|l|}{ Pré-operatório } & \multicolumn{2}{l|}{ Acompanhamento final } & \\
\hline & Média & Desvio padrão & Média & Desvio padrão & Significado \\
\hline Escala numérica de dor (dor nas pernas) & 7,37 & 0,889 & 1,57 & 1,765 & Valor de $p<0,0001$ \\
\hline Escala numérica de dor (dor nas costas) & 7,60 & 0,823 & 1,56 & 1,922 & Valor de $p<0,0001$ \\
\hline Escore ODI & 82,32 & 5,764 & 19,17 & 24,14 & Valor de $p<0,0001$ \\
\hline
\end{tabular}

Abreviatura: ODI, Oswestry disability index. 
mais espesso, formação de osteófitos, dura redundante devido a sedimentação espinhal degenerativa, e aumento da friabilidade dural foram descritos como fatores predisponentes que levaram ao aumento da incidência de ruptura dural na população idosa, segundo Yoshihara e Yoneoka. ${ }^{9}$

Analisando os dados da população octogenária no estudo Spine Patient Outcomes Research Trial (SPORT), os autores concluíram que não houve aumento significativo de complicação e mortalidade, após a cirurgia neste subconjunto da população. ${ }^{10}$

Não houve diferença significativa nas taxas de complicações entre os grupos somente de descompressão e de fusão em nosso estudo. Lee et al. ao reverem a literatura, não observaram nenhum efeito negativo nos resultados clínicos pela adição de fusão à cirurgia de descompressão na estenose espinhal. ${ }^{11}$

Foi relatada taxa de mortalidade perioperatória de 0,6 a $1,3 \%$ após cirurgia da coluna lombar. ${ }^{5,12}$ Não foram observadas mortes perioperatórias no presente estudo. Lee et al. ${ }^{11}$ revisaram a literatura sobre os resultados da cirurgia da coluna lombar em pacientes geriátricos e, observaram que não houve aumento significativo da mortalidade em indivíduos submetidos à cirurgia, em comparação com controles pareados por idade submetidos ao mesmo procedimento.

Em nosso estudo, o tempo médio de permanência hospitalar foi de 5,69 dias, sendo um pouco maior naqueles pacientes com fusão, em comparação aos que foram submetidos somente à descompressão. Os achados do nosso estudo foram comparáveis aos de Ragab et al., ${ }^{5}$ que observaram um tempo médio de internação de 4 dias no grupo descompressão e de 6 dias no de fusão.

Ao analisar os resultados de trinta e nove pacientes com idade superior a 80 anos, com estenose da coluna lombar, os pacientes descreveram redução significativa da dor nas costas e nas pernas, melhora na capacidade ambulatorial e no escore ODI. Ragab et al., ${ }^{5}$ em seu estudo com 118 pacientes com idade média de 74 anos, relataram uma satisfação geral em $68 \%$ dos pacientes, enquanto Shabat et al. $^{7}$ relataram uma taxa de satisfação de 76\%. Em nosso estudo, 76\% dos pacientes relataram estar satisfeitos com os resultados cirúrgicos.

É importante fazer uma distinção entre a idade cronológica e a idade funcional dos idosos e, assim, desafiar a crença convencional da idade como critério de exclusão para a cirurgia da coluna lombar. A idade não é uma contraindicação à cirurgia da coluna lombar, nem é um indicador de um evento catastrófico complicador em pacientes submetidos à cirurgia. No entanto, é necessário um monitoramento cuidadoso e uma maior vigilância, em relação à ocorrência de qualquer complicação perioperatória desfavorável neste subconjunto populacional de alto risco. ${ }^{13,14}$

O desenho retrospectivo do estudo é a principal limitação desta análise. Como os dados perioperatórios dos pacientes são anotados a partir de gráficos de registro de dados, alguns eventos ou complicações menores não registrados podem ter sido perdidos. Além disso, a falta de uma coorte comparativa de pacientes com idade inferior a 75 anos impediu a análise comparativa do estudo de coorte. $\mathrm{O}$ uso da entrevista telefônica como método de acompanhamento também foi uma limitação no estudo. $\mathrm{O}$ acompanhamento direto de pacientes não foi possível nessa população idosa, devido a questões relacionadas à ida até o hospital.

\section{Conclusão}

Com uma seleção cuidadosa dos pacientes e cuidados perioperatórios meticulosos, a cirurgia da coluna lombar é segura e eficaz na população idosa que não responde a medidas conservadoras. Os pacientes que tiveram maior tempo médio de internação hospitalar foi devido ao programa de reabilitação gradual e abrangente. A presença de comorbidades ou complicações perioperatórias menores, não aumentou a morbidade geral, nem afetou os resultados clínicos da cirurgia em nosso estudo. A idade por si só, não prediz o resultado das cirurgias da coluna lombar e, portanto, não deve ser usada como critério único no processo de tomada de decisão cirúrgica.

\section{Conflito de Interesses}

Os autores declaram não haver conflito de interesses.

\section{Referências}

1 Central Statistics Office. Ministry of Statistics and Programme implementation, Government of India. Elderly in India 2016. Disponível em: mospi.nic.in/sites/default/files/.../ElderlyinIndia_2016.pdf

2 Katz JN, Lipson SJ, Larson MG, McInnes JM, Fossel AH, Liang MH. The outcome of decompressive laminectomy for degenerative lumbar stenosis. J Bone Joint Surg Am 1991;73(06):809-816

3 Esses SI, Huler RJ. Indications for lumbar spine fusion in the adult. Clin Orthop Relat Res 1992;(279):87-100

4 Sanderson PL, Wood PL. Surgery for lumbar spinal stenosis in old people. J Bone Joint Surg Br 1993;75(03):393-397

5 Ragab AA, Fye MA, Bohlman HH. Surgery of the lumbar spine for spinal stenosis in 118 patients 70 years of age or older. Spine 2003;28(04):348-353

6 Panjabi MM. Clinical spinal instability and low back pain. J Electromyogr Kinesiol 2003;13(04):371-379

7 Shabat S, Arinzon Z, Folman Y, et al. Long-term outcome of decompressive surgery for lumbar spinal stenosis in octogenarians. Eur Spine J 2008;17(02):193-198

8 Smorgick Y, Baker KC, Herkowitz H, et al. Predisposing factors for dural tear in patients undergoing lumbar spine surgery. J Neurosurg Spine 2015;22(05):483-486

9 Yoshihara H, Yoneoka D. Incidental dural tear in spine surgery: analysis of a nationwide database. Eur Spine J 2014;23(02):389-394

10 Rihn JA, Hilibrand AS, Zhao W, et al. Effectiveness of surgery for lumbar stenosis and degenerative spondylolisthesis in the octogenarian population: analysis of the Spine Patient Outcomes Research Trial (SPORT) data. J Bone Joint Surg Am 2015;97(03):177-185

11 Lee JY, Moon SH, Suh BK, Yang MH, Park MS. Outcome and Complications in Surgical Treatment of Lumbar Stenosis or Spondylolisthesis in Geriatric Patients. Yonsei Med J 2015;56(05):1199-1205

12 Oldridge NB, Yuan Z, Stoll JE, Rimm AR. Lumbar spine surgery and mortality among Medicare beneficiaries, 1986. Am J Public Health 1994;84(08):1292-1298

13 Jakola AS, Sørlie A, Gulati S, Nygaard OP, Lydersen S, Solberg T. Clinical outcomes and safety assessment in elderly patients undergoing decompressive laminectomy for lumbar spinal stenosis: a prospective study. BMC Surg 2010;10:34

14 Arinzon ZH, Fredman B, Zohar E, et al. Surgical management of spinal stenosis: a comparison of immediate and long term outcome in two geriatric patient populations. Arch Gerontol Geriatr 2003;36(03):273-279 